\title{
Efficacy of Tranexamic Acid on Blood Loss in Thoracolumbar Spinal Fusion Surgery
}

\author{
Keskin Emrah ${ }^{1}$, Aydin Hasan Ali $^{1}$, Ozgen Utku ${ }^{2}$, Kalayci Murat ${ }^{1}$, Simsek Kenan ${ }^{1}$ and Baklaci Deniz ${ }^{3}$ \\ ${ }^{1}$ Department of Neurosurgery, Bulent Ecevit University Medical Faculty, Zonguldak, Turkey \\ ${ }^{2}$ Department of Neurosurgery, Ataturk State Hospital, Zonguldak, Turkey \\ ${ }^{3}$ Department of Otorhinolaryngology, Bülent Ecevit University Medical Faculty, Zonguldak, Turkey
}

\begin{abstract}
Objective: To investigate the effectiveness of topical tranexamic acid (TXA) use on bleeding and drain withdrawal time in adult patients undergoing thoracolumbar fusion surgery.

Study Design: Cross-sectional comparative study.

Place and Duration of Study: Zonguldak Bülent Ecevit University Hospital, Zonguldak, Turkey, from January 2019 to August 2020.

Methodology: The data of patients, who received thoracic and/or lumbar fusion surgery, were evaluated. The patients were divided into two groups as Group A (30 patients) in which $1 \mathrm{~g}$ topical TXA was applied to the surgical site at the end of the surgery; and Group B (30 patients) who did not receive this medication.

Results: The body mass index (BMI) and the patients' demographic factors were similar in both the groups. There were no statistically significant differences between the two groups in terms of the surgical time and level, perioperative ES and FFP transfusion rates $(p>0.05)$. While the amount of perioperative bleeding was almost the same $(650 \mathrm{~mL}, p=$ 0.778), the amount of postoperative bleeding was lower in Group A (138.1 $\pm 55.6 \mathrm{~mL}$ ) in comparison to Group B (230.3 $\pm 65.4 \mathrm{~mL}$ ). Postoperative ES transfusion was performed on two patients in Group A (6.7\%) and six patients in Group B (30\%) $(p=0.02)$. Accordingly, the mean drain withdrawal time in Group A was 3 (2.8-3.0); days, and in Group B, this time was $6(5.0-6.0)$ days $(p<0.001)$. Systemic side effects, especially thromboembolism, were not observed in any of the patients.
\end{abstract}

Conclusion: The topical use of TXA in spinal fusion surgery reduced perioperative blood loss.

Key Words: Blood loss, Thoracolumbar spinal surgery, Tranexamic acid.

How to cite this article: Emrah K, Ali AH, Utku O, Murat K, Kenan S, Deniz B. Efficacy of Tranexamic Acid on Blood Loss in Thoracolumbar Spinal Fusion Surgery. J Coll Physicians Surg Pak 2021; 31(12):1449-1454.

\section{INTRODUCTION}

Especially in the last 50 years, spinal fusion surgery (SFS) has been used frequently in many different pathologies, especially degenerative diseases. SFS is a staged surgery (opening, decompression, instrumentation and closure), takes a long time and requires a large incision area. Therefore, it may cause significant blood losses that require blood transfusion. ${ }^{1}$ Thoracolumbar spinal fusion surgery (TLSFS) in adults causes severe blood loss and often requires blood transfusion due to this condition. ${ }^{2,3}$

Correspondence to: Dr. Keskin Emrah, Department of Neurosurgery, Bülent Ecevit University Medical Faculty, Zonguldak, Turkey, Güney District Barış Street, Rüya Buildings, 2/6, Kozlu-Zonguldak, Turkey

E-mail:drkeskinemrah@gmail.com

Received: October 01, 2020; Revised: March 19, 2021; Accepted: May 17, 2021

DOI: https://doi.org/10.29271/jcpsp.2021.12.1449
Many anatomicalfactors may increasetherisk of bleeding, especially in the Batson's venous plexus, paraspinal muscles and the vessels that feed the joints of the spine. ${ }^{4,5}$ Prolonged operation time leads to frequent allogeneic blood transfusions, which may increase the risk of allergic reactions, infections and blood incompatibility. ${ }^{6}$ In the literature, several methods have been introduced to reduce perioperative blood loss and transfusion requirements. These methods include hypotensive anesthesia, normovolemic hemodilution and perioperative blood transfusion. ${ }^{1,7,8}$ Although various treatment methods have been recommended, blood loss remains a significant issue in spinal surgery.

Antifibrinolytic agents, such as anti-hemostatic agents, are of great interest among surgeons in terms of reducing perioperative blood loss. Since tranexamic acid (TXA) had first entered clinical practice in women with severe menstrual bleeding in the mid-1900s, the results from numerous clinical studies have confirmed its effectiveness in reducing the need for transfusion due to intraoperative bleeding. ${ }^{9}$ TXA is an analogue of the amino acid lysine (synthetic) and an antifibrinolytic agent that can 
inhibit both plasminogen activation and the effect of plasmin. ${ }^{10}$ It provides an antifibrinolytic effect by preventing the breakdown of a clot that is formed without forming a new clot. It reduces the conversion of plasminogen to plasmin. It does this by binding to lysine binding sites on the plasminogen surface and preventing plasminogen from binding to lysine on fibrin. At the same time, it protects the clot formed by preventing binding to fibrin by creating a blockage in the lysine binding sites of the circulating plasmin. ${ }^{9,10}$ TXA also preserves platelet function through plasmin inhibition. ${ }^{8}$ It is used not only systemically but also topically and locally. However, systemic side effects are the main reason why surgeons hesitate to use anti-fibrinolytic agents systemically. ${ }^{8}$ It TXA application is not a newly discovered method, and its use as a routine part of any surgery has not been widely accepted yet. When applied topically, despite its low systemic absorption, its beneficial effect in reducing bleeding is observed not only in normally healthy individuals but also in patients receiving hemorrhagic diathesis and preoperative anticoagulant drugs. It has been reported that positive results have been obtained regarding the topical use of TXA in cardiovascular surgery, orthopedics, and plastic surgery operations. 8 ,11-13

The aim of this study, was to investigate the efficacy of perioperative topical TXA use on postoperative blood loss and drain withdrawal time in adult patients undergoing TLSFS.

\section{METHODOLOGY}

This retrospective study was conducted with 60 patients, who underwent thoracic and/or lumbar fusion surgery in 2019 and 2020 at the Department of Neurosurgery, Zonguldak Bülent Ecevit University Faculty of Medicine. It was initiated by obtaining the approval of the local Ethics Committee of the Hospital with referral centre (Decree No. 2020/17). The sample consisted of patients regardless of gender, who had complaints that persisted despite the use of non-surgical methods and underwent thoracic and/or lumbar SFS (spinal stenosis, spondylolisthesis, etc.). The inclusion criteria were as patients $>18$ years of age, without coagulation or hemoglobin dysfunction and undergoing elective posterior TLSFS (one or more level). The exclusion criterion was patients with missing data, coagulation disorders, history of renal failure or kidney transplant, hepatorenal or cardiopulmonary dysfunction history, arterial thromboembolic event within the past year (deep vein thrombosis, ischemic heart disease, pulmonary embolism, strokes) and patients with leakage of cerebrospinal fluid (CSF) during or after surgery. All surgical procedures were performed by the same surgeon (KE).

All operations were performed in the prone position under general anesthesia, and intravenous (iv) prophylactic antibiotic treatment was administered with cefazolin. Cefazolin was applied as $1 \times 2 \mathrm{~g}$ iv one hour before surgery and $3 \times 1 \mathrm{~g}$ iv (intravenously) at a maximum of three doses, eight hours after surgery. The surgical level was determined by placing a metal marker on the surface under the guidance of a C-arm fluoros- cope. After a longitudinal skin incision was made along the midline, bleeding was controlled with electrocautery at all stages of the surgery. The paravertebral muscle fascia and muscles were bluntly dissected with the aid of monopolar cautery, and retraction was undertaken using the same retractor in both the groups. After reaching the surgical site, pedicle screws were successfully inserted using a C-arm fluoroscope. Laminectomy was performed using the Kerrison rongeur and/or a motor. Previously beveled longitudinal rods were installed on both sides and then interconnected. Then $1 \mathrm{~g}$ TXA (Transamin ${ }^{\circledR} 250 \mathrm{mg}$; Istanbul; 5-mL vials) was poured into the surgical side and then left until it was absorbed (3-5 minutes on average). After bilateral posterolateral bone grafting and negative pressure drainage tube placement, the layers were closed in the anatomical plane.

Intraoperative bleeding was determined using gauze, blood in the aspiration bag, and all irrigation fluids (including saline). Blood transfusion was performed in patients estimated to have an intraoperative blood loss of greater than $700 \mathrm{~mL}$ or $<7.0$ $\mathrm{g} / \mathrm{dL}$ postoperative hemoglobin concentration, and in those who were not stable despite fluid resuscitation and erythrocyte replacement, at the discretion of the anesthesiologist. In the postoperative blood transfusion protocol, if the patient had hypovolemia findings (pallor, sweating, tachycardia, hypotension) and an accompanying hemoglobin value between 7.0 and $8.0 \mathrm{~g} / \mathrm{dL}$, or if the hemoglobin value below $7.0 \mathrm{~g} / \mathrm{dL}$, erythrocyte suspension transfusion (ES) was performed. Fresh frozen plasma (FFP, $200 \mathrm{~mL}$ each) was applied on the patients with intraoperative bleeding with an international normalised ratio (INR) of $>1.5$ or prolonged prothrombin time (PT) and activated partial thromboplastin time (aPTT).

As a routine practice at this clinic, the amount of bleeding in the drains was recorded three times a day. If the amount of bleeding from the drains during the shift periods was less than $50 \mathrm{~mL}$, the drains were removed, and the patient was discharged on the same day. From the hospital file records, the patients' age, gender, weight, height, body mass index (BMI), amount of bleeding during surgery, duration of surgery, intraoperative and postoperative ES and FFP transfusions, number of surgical segments, and time to drain tube withdrawal were obtained and examined.

The data were analysed using the statistical package for the social sciences version 22.0. The categorical data are presented given as frequencies and percentages, while the quantitative data are presented as mean \pm SD and median (IQR: Q1-Q3). For normal distribution assumptions, ShapiroWilk test was used. The Skewness and Kurtosis coefficients and distribution in q-q plot graphs were checked. Independent sample t-test or Mann-Whitney U-test was used to determine the significant differences between groups depending upon normality of variables. The differences between the groups for the categorical variables were evaluated using Chi-squared and Likelihood ratio tests. A p-value of $<0.05$ was accepted as statistically significant. 
Table I: Clinical information for the 2 groups of patients.

\begin{tabular}{|l|l|c|c|c|c|c|c|}
\hline Groups & & Male & Female & Age & BMI & Surgical segments & DM* \\
\hline A & 30 & $14(46.7 \%)$ & $16(53.3 \%)$ & $63.0(59.0-68.3)$ & $29.6(27.2-31.4)$ & $3.0(2.0-4.0)$ & $10(33.3 \%)$ \\
\hline B & 30 & $13(43.3 \%)$ & $17(56.7 \%)$ & $63.5(53.567 .0)$ & $30.0(28.4-33.8)$ & $3.0(2.0-4.0)$ & $13(43.3 \%)$ \\
\hline P & \multicolumn{2}{|c|}{0.795} & 0.358 & 0.246 & 0.693 & 0.426 \\
\hline
\end{tabular}

between the sex, DM and tranexamic acid groups. Age, BMI and Surgical Segments are presented as medians (Q1-Q3) due to nonparametric distribution.

Table II: Intraoperative and postoperative measures.

\begin{tabular}{|l|c|c|c|}
\hline Measures & Group A & Group B & p \\
\hline $\begin{array}{l}\text { Surgical time, } \\
\text { min }\end{array}$ & $\begin{array}{c}210.0 \\
(176.3-243.8)\end{array}$ & $\begin{array}{c}185.0 \\
(150.0-222.5)\end{array}$ & $0.154^{*}$ \\
\hline $\begin{array}{l}\text { Estimated perioperative blood loss, } \\
\mathrm{mL}\end{array}$ & $\begin{array}{c}650.0 \\
(400.0-1000.0)\end{array}$ & $\begin{array}{c}650.0 \\
(487.5-812.5)\end{array}$ & $0.778^{*}$ \\
\hline $\begin{array}{l}\text { Perioperative number (\%) receiving } \\
\text { fresh frozen plasma (FFP) }\end{array}$ & $4(13.3 \%)$ & $8(26.7 \%)$ & $0.197^{\dagger}$ \\
\hline $\begin{array}{l}\text { Perioperative number (\%) receiving } \\
\text { blood transfusion (ES) }\end{array}$ & $9(30 \%)$ & $14(46.7 \%)$ & $0.184^{\dagger}$ \\
\hline $\begin{array}{l}\text { Postoperative number (\%) receiving } \\
\text { blood transfusion (ES) }\end{array}$ & $2(6.7 \%)$ & $9(30 \%)$ & $0.020^{\dagger}$ \\
\hline $\begin{array}{l}\text { EBL presented as medians (Q1-Q3) due to nonparametric distribution. }{ }^{\dagger} \text { The difference was evaluated by } \\
\text { Mann-Whitney U test. }{ }^{\dagger} \text { Chi-squared test. }\end{array}$
\end{tabular}

Table III: Comparison of the effectiveness of the tranexamic acid group and control group regardless of spine level.

\begin{tabular}{|c|c|c|c|}
\hline Measures & $\underset{n=30}{\text { Group } A}$ & $\underset{n=30}{\text { Group } B}$ & p-value \\
\hline Time to withdraw the drain tube $(\mathrm{mL})$ & $2.9 \pm 0.7$ & $5.5 \pm 1.2$ & $<0.001$ \\
\hline Overall post-op drain mean $(\mathrm{mL})$ & $138.1 \pm 55.6$ & $230.3 \pm 65.4$ & $<0.001$ \\
\hline \multicolumn{4}{|l|}{ Postoperative day } \\
\hline 2 & $7(23.3 \%)$ & $1(3.3 \%)$ & \\
\hline 3 & $19(63.3 \%)$ & $2(6.7 \%)$ & \\
\hline 4 & $3(10 \%)$ & $1(3.3 \%)$ & $<0.001$ \\
\hline 5 & $1(3.3 \%)$ & $6(20 \%)$ & \\
\hline 6 & 0 & $16(53.3 \%)$ & \\
\hline 7 & 0 & $4(13.3 \%)$ & \\
\hline
\end{tabular}

Independent-samples t-test was run for time to withdraw the drain tube, overall post op drain mean difference according to the tranexamic acid groups

Likelihood ratio test was used for postoperative day.

Table IV: Comparison of the effectiveness of the tranexamic acid group and the control group on multiple spinal levels.

\begin{tabular}{|l|l|c|c|c|}
\hline Groups & & $\begin{array}{c}\text { Time to withdraw } \\
\text { the drain tube } \\
\text { (day) }\end{array}$ & $\begin{array}{c}\text { Post-op drain } \\
\text { first day mean } \\
\text { (mL) }\end{array}$ & $\begin{array}{c}\text { Overall post-op } \\
\text { drain mean }(\mathbf{m L})\end{array}$ \\
\hline Group A & 26 & $2.9 \pm 0.7$ & $216.9 \pm 62.5$ & $136.0 \pm 53.5$ \\
\hline Group B & 26 & $5.9 \pm 0.8$ & $304.6 \pm 67$ & $241.6 \pm 51.8$ \\
\hline $\mathrm{p}$ & $<0.001$ & $<0.001$ & $<0.001$ \\
\hline
\end{tabular}

\section{RESULTS}

The patients were divided into two groups as Group A (30 patients), in which $1 \mathrm{~g}$ topical TXA was applied to the surgical site at the end of the surgery; and Group B (30 patients) who did not receive TXA. There was no significant difference between the two groups in terms of their general characteristics (age, gender and BMI) and comorbidities ( $p>0.05$, Table I). The surgical level distribution of the cases in Group $A$ were as follows: single segment in 4 cases ( $13.3 \%), 2$ segments in 8 cases $(26.7 \%), 3$ segments in 7 cases $(23.3 \%), 4$ segments in 6 cases $(20 \%)$ and 5 or more segments in 5 cases (16.7\%); whereas, the median number of surgical segments was 3.0 segments. The surgical level distribution of the patients in Group B were as follows: single segment in 4 cases $(13.3 \%), 2$ segments in 10 cases (33.3\%), 3 segments in 5 cases $(16.7 \%), 4$ segments in 8 cases $(26.7 \%)$, and 5 or more segments in 3 cases (10\%); whereas, the median number of surgical segments in this group was 3.0 (Table I).

There was no significant difference between the groups in terms of their surgical levels ( $p=0.693$, Table I). The perioperative bleeding amount was equal in both the groups $(650$ (400.0-1000.0) mL vs. 650 (487.5-812.5) mL, respectively; $p=0.778$, Table II), and there was no significant difference between the two groups in terms of operation time (210 (176.3-243.8) min vs. 185 (150.0-222.5) minutes, respectively; $p=0.154$, Table II). Regardless of the spinal level, in Group $A$, the amount of postoperative bleeding was lower in the first 24 hours $(216 \pm 62.5 \mathrm{~mL}$ and $292 \pm 82.5 \mathrm{~mL}$, respectively, $\mathrm{p}<0.001$, Table III) and on the following days (138.1 $\pm 55.6 \mathrm{~mL}, 230.3 \pm 65.4 \mathrm{~mL}, \mathrm{p}<0.001$, Table III) in comparison to Group B, and this difference was statistically significant. Additionally, in Group A, the amount of postoperative bleeding in the first 24 hours $(216.9 \pm 62.5 \mathrm{~mL}, 304.6 \pm$ $67 \mathrm{~mL}$, respectively, $\mathrm{p}<0.001$, Table IV) and on the following days $(136.0 \pm 53.5 \mathrm{~mL}, 241.6 \pm 51.8 \mathrm{~mL}$, respectively, $p<0.001$, Table IV) was significantly lower on multiple spinal levels in comparison to Group B. The drain was removed on the postoperative approximately in 3 days (2.9 $\pm 0.7)$ in Group A and 6 days $(5.5 \pm 1.2)$ in Group B (Table III).

While FFP was given to 4 (13.3\%) patients, and ES was given to $9(30 \%)$ patients perioperatively in Group A, 8 (26.7\%) patients were given FFP, and 14 (46.7\%) patients were given ES in Group B. No difference was observed between the two groups in relation to their perioperative FFP and ES transfusion rates $(p=0.197, p=0.184$, respectively, Table II). On the other hand, $2(6.7 \%)$ patients were given ES in the postoperative period in Group A, and 9 (30\%) patients were given ES in Group B. As a result, a significant difference was found in favour of the TXA group in terms of the need for ES in the postoperative period ( $p=0.02$, Table II).

All these data showed that topical TXA (Group A) application, 
both reduced postoperative bleeding and enabled the earlier withdrawal of the drain ( $p<0.001$, Table IV). Accordingly, the hospital stay was shorter in the TXA group due to the patients being discharged on the day the drains were withdrawn. There was no discharge, infection or other complication from the incision site in either group. At the end of the $48^{\text {th }}$ postoperative hour, deep vein thrombosis was not observed in either group.

\section{DISCUSSION}

SFS is currently the most common surgical procedure for the spine. However, it can cause serious blood loss and may require longer operation times. ${ }^{14}$ Various treatment procedures are used in spinal surgery, including controlled hypotension, blood substitutes and pharmacological therapies to reduce perioperative blood loss. ${ }^{15,16}$ Additionally, the use of antifibrinolytic drugs in many types of surgery has been increasing in recent years. Because of the high risk of graft occlusion due to the use of systemic aprotinin in coronary bypass surgery, for the first time, Tatar et al. tried the use of a topical antifibronolytic. ${ }^{17}$ As a result, they showed that the topical use of aprotinin reduced postoperative blood loss and the need for transfusions. Although the reliability of antifibrinolytic drugs has been questioned for a while due to aprotinin-related deaths, EACA and TXA are the more reliable drugs in this class. TXA is widely used both iv and topically in cardiovascular and orthopedic surgeries (hip and knee arthroplasty). However, its use in neurosurgery is uncommon. ${ }^{10,11}$ There are a limited number of studies on the use of TXA in spinal surgery. In this study, the authors investigated the effects of topical TXA use on postoperative bleeding regardless of spinal surgery level.

There are few studies showing that TXA can reduce blood loss by inhibiting fibrinolytic activity in spinal deformity and fusion surgery. Most studies have shown that TXA reduces postoperative bleeding with or without statistical significance. ${ }^{18,19}$ Elwatidy et al. showed in their double-blind, randomised and placebo-controlled study that high-dose iv TXA reduced blood loss by $49 \%$, although the difference was not statistically significant, in pediatric and adult spinal surgery. ${ }^{18}$ In their retrospective examination of idiopathic scoliosis patients, Berney et al. found that intraoperative bleeding was reduced by approximately $50 \%$ in those receiving iv TXA. ${ }^{19}$ However, in their study, there was no statistically significant difference between the groups in terms of hospital stay. In this study, unlike the aforementioned studies, postoperative bleeding was reduced by approximately $60 \%$ in Group A in comparison to Group B ( $p$ $<0.001$ ), and the mean time of drain withdrawal was approximately 3 days ( $p<0.001$, Table III). As a result of this, the hospital stay was shorter in Group A.

Side effects related to TXA include acute gastrointestinal discomfort (nausea, vomiting and diarrhea), visual disturbances (blurred vision, change in color perception), acute renal cortical necrosis with oliguria and renal failure, thromboembolic events (deep vein thrombosis, pulmonary embolism, DIC) and convulsion. Whether or not TXA has a thromboembolic side effect is still debated. Many studies on TXA (cardiovascular and orthopedic surgery) did not show an increase in the incidence of thrombosis in comparison to other groups (control or placebo)., ${ }^{9,18}$ However, the exclusion criteria in these studies were an important factor affecting this result. High-risk patients were excluded, especially in studies conducted in orthopedic surgery. For example, excluded patients included those with a history of cardiovascular disease, thromboembolic events or kidney failure. 6,20 Pernik et al. observed that iv TXA not only provided a protective effect against perioperative blood loss in patients who had 4 or more SFS, but it also did not increase the risk of thrombotic events. ${ }^{20}$ Similarly, in this study, topical TXA reduced postoperative bleeding, and no thrombotic side effects were observed in 52 patients who had multiple-level TLSFS (Table IV).

TXA can be administered with iv, topically, locally or orally. In literature, most studies have investigated the effects of oral or iv TXA on perioperative bleeding or postoperative transfusion requirements. ${ }^{6,15,21,22}$ In one study, it was observed that iv TXA reduced perioperative blood loss in patients with multi-level thoracic spinal stenosis without any systemic side effects. ${ }^{23}$ In another study, the effects of iv and oral TXA in reducing blood loss in TLSFS were found to be equal. ${ }^{15}$ In the study of Kumar et al., where the authors examined the effectiveness of the intravenous and local use of TXA in elective spinal surgery, it was seen that these were equally effective in reducing perioperative blood loss. ${ }^{24}$ In comparison to knee arthroplasty surgery, spinal surgery has more extensive dissections and involves a larger operation field. Thus, there is a tendency to prefer iv over topical administration of TXA. ${ }^{6}$ Topical TXA can be considered as a more reliable and effective method as it acts directly at the active bleeding sites and has fewer systemic side effects. However, there are few studies that have investigated the effects of topically applied TXA. ${ }^{21,22}$ Liang et al. showed that the use of intraoperative topical TXA (to the lumbar spine surgery site with an absorbable gelatin sponge) shortened the duration of hospital stay due to reduced postoperative blood loss. ${ }^{21}$ Ren et al. reported that the use of topical TXA at the end of surgery not only reduced postoperative blood loss but also did not increase the risk of developing thromboembolic complications and shortened the duration of hospital stay. ${ }^{22}$ On the other hand, in this study, TXA was applied topically instead of iv injection in TLSFS surgery. Patients in Group A did not have any systemic or local side effects and that the postoperative drain withdrawal time was shorter in Group A than Group B ( $p<0.001)$. Additionally, the postoperative ES transfusion rate $(p=0.020$; Table II) and postoperative bleeding amount were lower in Group $A$ in comparison to Group B ( $p<0.001$, Table IV). 
Limitations of this study also need to be addressed. There were only 30 cases in the TXA group, and the whole sample size was relatively small. As the number of cases receiving TLSFS was low in this study, it was not possible to compare the effects of topical TXA application on bleeding between those who received single-level TLSFS and those who received multi-level TLSFS. Further randomised controlled clinical studies with larger patient populations are needed to support the positive effects of TXA on perioperative and postoperative blood loss.

\section{CONCLUSION}

Intraoperative use of TXA reduces the need for postoperative ES and the time to drain tube withdrawal; thus, shortening the postoperative hospital stay of patients.

\section{ACKNOWLEDGMENT:}

We thank Cansu Başar for her assistance in the statistical analysis of the study.

\section{ETHICAL APPROVAL:}

This study was conducted in compliance with the ethical principles of the Declaration of Helsinki, and it was approved by the local Institutional Review Board (Number: 2020/17).

\section{PATIENTS' CONSENT:}

Since this was designed as a retrospective study, the data were collected from the hospital archives after the approval of the Ethics Committee.

\section{CONFLICT OF INTEREST:}

The authors declared no conflict of interest.

\section{AUTHORS' CONTRIBUTION:}

KE, SK: Concept and design of study, data collection and analysis.

OU: Test performance, draft formation and review.

$\mathrm{KE}, \mathrm{KM}, \mathrm{AHA}$ : Analysis, review and final approval of draft.

$\mathrm{KE}$ : Data collection, analysis and results compilation.BD: Concept and design of study, analysis and draft formation.

\section{REFERENCES}

1. Hu SS. Blood loss in adult spinal surgery. Eur Spine J 2004; 13(Suppl 1):3-5. doi:10.1007/s00586-004-0753-x.

2. Nuttall GA, Horlocker TT, Santrach PJ, Oliver WC, Dekutoski MB, Bryant S. Predictors of blood transfusions in spinal instrumentation and fusion surgery. Spine 2000; 25(5): 596-601. doi:10.1097/00007632-200003010-00010

3. Behrman MJ, Keim HA. Perioperative red blood cell salvage in spine surgery. A prospective analysis. Clin Orthop Relat Res 1992; 278:51-7.

4. Nagabhushan RM, Shetty AP, Dumpa SR, Subramanian B, Kanna RM, Shanmuganathan R. Effectiveness and safety of batroxobin, tranexamic acid and a combination in reduction of blood loss in lumbar spinal fusion surgery. Spine 2018; 43(5):E267-E73. doi:10.1097/BRS.0000000000002315.

5. Lee DY, Park YJ, Song SY, Jeong ST, Kim DH. Risk factors for posterior cage migration after lumbar interbody fusion surgery. Asian Spine J 2018; 12(1):59-68. doi:10.4184/ asj.2018.12.1.59.

6. Yu CC, Kadri O, Kadado A, Buraimoh M, Pawloski J, Bartol S, et al. Intravenous and oral tranexamic acid are equivalent at reducing blood loss in thoracolumbar spinal fusion: $A$ prospective randomized trial. Spine 2019; 44(11):755-61. doi:10.1097/BRS.0000000000002954.

7. Müslüman AM, Yılmaz A, Cansever $T$, Çavuşoğlu H, Çolak I, Genç HA, et al. Posterior lumbar interbody fusion versus posterolateral fusion with instrumentation in the treatment of low-grade isthmic spondylolisthesis: midterm clinical outcomes. J Neurosurg Spine 2011; 14(4):488-96. doi:10.3171/2010.11.SPINE10281.

8. Neilipovitz DT. Tranexamic acid for major spinal surgery. Eur Spine J 2004; 13(Suppl 1):62-5. doi:10.1007/s005 86-004-0716-2.

9. Tengborn L, Blombäck M, Berntorp E. Tranexamic acid-an old drug still going strong and making a revival. Thromb Res 2015; 135(2):231-42. doi.org/10.1016/j.thromres. 2014.11.012.

10. Mannucci PM. Hemostatic drugs. N Engl J Med 1998; 339(4):245-5I. doi:10.1056/NEJM199807233390407.

11. Chaudhary FA, Pervaz Z, Ilyas S, Niaz MN. Topical use of tranexamic acid in open heart surgery. J Pak Med Assoc 2018; 68(4):538-542.

12. Melvin JS, Stryker LS, Sierra RJ. Tranexamic acid in hip and knee arthroplasty. J Am Acad Orthop Surg 2015; 23 : 732-40. doi: 10.5435/JAAOS-D-14-00223.

13. Fayman $M$, Beeton A, Potgieter $E$, Ndou R, Mazengenya P. Efficacy of topical tranexamic acid (cyclokapron) in "wet" field infiltration with dilute local anaesthetic solutions in plastic surgery. Aesthetic Plast Surg 2021; 45(1):332-9. doi.org/10.1007/s00586-004-0716-2.

14. Hosseini P, Mundis GM, Eastlack RK, Eastlack RK, Bagheri R, Vargas $E$, et al. Preliminary results of anterior lumbar interbody fusion, anterior column realignment for the treatment of sagittal malalignment. Neurosurg Focus 2017; 43(6): E6 doi:10.3171/2017.8.FOCUS17423.

15. Yang B, Li H, Wang D, He X, Zhang C, Yang P. Systematic review and meta-analysis of perioperative intravenous tranexamic acid use in spinal surgery. PLoS One 2013; 8(2):e55436. doi:10.1371/journal. pone.0055436.

16. Peters A, Verma K, Slobodyanyuk K, Cheriyan T, Hoelscher C, Schwab F, et al. Antifibrinolytics reduce blood loss in adult spinal deformity surgery: A prospective, randomised controlled trial. Spine 2015; 40(8):E443-9. doi:10.1097/ BRS.0000000000000799.

17. Tatar H, Ciçek S, Demirkiliç U, Ozal E, Süer H, Oztürk O, et al. Topical use of aprotinin in open heart operations. Ann Thorac Surg 1993; 55:659-661. doi.org/10.1016/00034975(93)90271-I.

18. Elwatidy S, Jamjoom Z, Elgamal E, Zakaria A, Turkistani A, El-Dawlatly A. Efficacy and safety of prophylactic large dose of tranexamic acid in spine surgery: A prospective, randomised, double-blind, placebo-controlled study. Spine 2008; 33(24):2577-80. doi:10.1097/BRS.0b013e318188 b9c5. 
19. Berney MJ, Dawson PH, Phillips M, Lui DF, Connolly P. Eliminating the use of allogeneic blood products in adolescent idiopathic scoliosis surgery. Eur J Orthop Surg Traumatol 2015; 25(Suppl 1):219-23. doi:10.1007/s00 590-015-16 24-3.

20. Pernik MN, Dosselman LJ, Aoun SG, Walker AD, Hall K, Reyes $V P$, et al. The effectiveness of tranexamic acid on operative and perioperative blood loss in long-segment spinal fusions: A consecutive series of 119 primary procedures. J Neurosurg Spine 2020:1-7. doi:10.3171/ 2019.11.SPINE191174.

21. Liang J, Liu H, Huang $X$, Xiong $W$, Zhao $H$, Chua S, et al. Using tranexamic acid soaked absorbable gelatin sponge following complex posterior lumbar spine surgery: A randomised control trial. Clin Neurol Neurosurg 2016; 147:110-14. doi:10.1016/j.clineuro.2016.06.001.

22. Ren Z, Li S, Sheng L, Zhuang Q, Li Z, Xu D, et al. Topical use of tranexamic acid can effectively decrease hidden blood loss during posterior lumbar spinal fusion surgery: A retrospective study. Medicine 2017; 96(42):e8233. doi:10. 1097/MD.0000000000008233.

23. Xue P, Yang J, Xu X, Liu T, Huang Y, Qiao F, et al. The efficacy and safety of tranexamic acid in reducing perioperative blood loss in patients with multilevel thoracic spinal stenosis: A retrospective observational study. Medicine 2018; 97(50):e13643.doi: 10.1097/MD.00000000000 13643.

24. Arun-Kumar V, Naresh-Babu J. There a role for preoperative local infiltration of tranexamic acid in elective spine surgery? A prospective randomised controlled trial analyzing the efficacy of intravenous, local infiltration, and topical administration of tranexamic acid. Global Spine J 2021; 11(1):21-27. doi: 10.1177/2192568219888446. 